\title{
EASE OF INDIVIDUAL JUDGMENT-PROCESSES IN RELATION TO POLARIZATION OF ATTI- TUDES IN THE CULTURE
}

\author{
Department of Psychology, Yale University
}

\section{Charles E. Osgood*}

\section{A. Introduction}

The term "polarization" has been coming into frequent use in the social sciences. The term has appeared in the writings of political scientists (3), psychologists (1, pp. 160,303), (5, pp. 18-19), and sociologists (10, pp. 299-301). Although the term has been used with slightly varying connotations, the common essence of meaning seems to be action or thought which occurs in extremes.

In individual behavior it has signified the tendency for a person to think or act on what might be called an "all or none" basis (9). For example, the Lynds, in Middletown in Transition (8, p. 498), describe this polarizing tendency without employing the actual term when they say, "With its characteristic proclivity for resolving issues into stark blacks and whites and personalizing each within the manageable compass of a devil or a savior, business leaders in Middletown see in Roosevelt all that they are against. . . ." Polarization has been shown to be closely related to emotion, as for example, in the highly affective reaction given today to such stereotypes as communist and fascist.

The term has also been used in a more sociological sense, as the tendency for behavior in a group or culture to be restricted to a small portion of a possible range of response. The latter use of the term implies that the majority of the individuals forming the group fall, as it were, on one extreme of a distribution, yielding the characteristic J-curve of conforming behavior (2). The extreme and highly uniform attitudes displayed by an aroused populace in wartime constitute good illustrations of polarization in this sociological sense.

*From the Department of Psychology, Dartmouth College. The author wishes to acknowledge the assistance of Dr. Ross Stagner in the treatment of the data and the preparation of the manuscript for publication. 
Much of the research of which this paper is a part ${ }^{1}$ has been sociological in nature. That is, the results have been in terms of distributions of a large group of people. Therefore these results have been interpreted largely in cultural concepts. Although it is obvious that many relationships exist between such sociological patterns and individual thinking, any theories as to the nature of individual thinking based on such data must be rather inferential.

It may be seen, then, that in our work, as well as in much of psychosociological research, there has been a gap between individual and group data which has required inferential bridging. The purpose of the present study is to analyze one possible relationship between cultural stereotypes and individual thinking. Polarization is the possible relationship chosen for study. Ease of judgment, as measured by speed of decision, is used as an individual criterion. The problem may be worded as follows: if a representative sample of a culture shows polarization of certain patterns of thinking (e.g., the strength or weakness of dictators, the value or worthlessness of human beings), will individuals drawn from that culture exhibit greater ease in making judgments on these polarized patterns than on others?

\section{B. Procedure}

The materials for the present experiment were drawn from the studies mentioned above. Test forms were sent to various adult groups in the country on which they were to make judgments on the relationship between various concepts and gradients. ${ }^{2}$ An item of these group tests appeared as follows:

FIGHTING valuable : : : : : : worthless

with the subject instructed to check on the gradient to indicate his judgment. Analysis of the group returns indicated that certain items could be called polarized, i.e., a large proportion of the responses tended to pile up on one end of the gradient. Other items, on the contrary, were designated as spread items, i.e., the responses were distributed rather evenly over the gradient.

\footnotetext{
${ }^{1}$ Stagner, R., \& Osgood, C. E. Studies in nationalistic frames of reference as infuenced by the European War, in progress. Also, the companion paper to this, Stagner, R., \& Osgood, C. E. An experimental analysis of a nationalistic frame of reference.

${ }^{2}$ For a more complete discussion of the use of these terms see (7) and (11).
} 


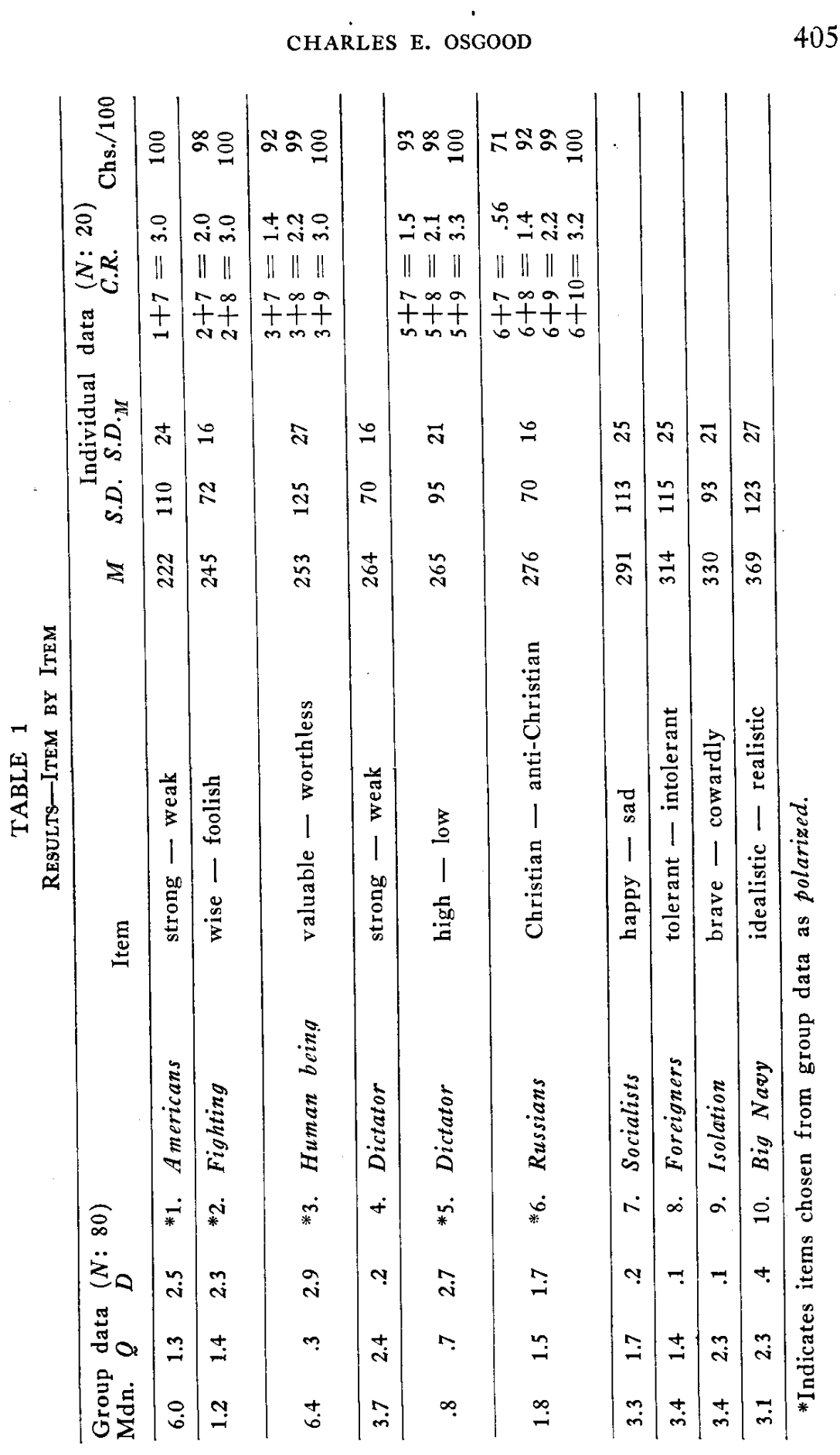


From the returns of a typical group of 80 adults, five items which showed a high degree of polarization and five items which were spread were picked for use in this present experiment. These are given in Table 1. The polarized items are shown by asterisks. Although the items are ordered in the table according to mean time of response, in actual presentation they appeared in alternation, odd being spread and even polarized.

In Table 1 are given medians, differences of medians from the actual mid-point of scales $(D)$, and $Q$ values, as determined graphically from cumulative frequency curves, for each of the items. These figures summarize the group data. It will be noted that for the polarized items the medians tend toward one extreme or the other ( 1 or 7 ) and the $Q$ 's are small. For spread items the medians hover close about the middle of the gradients (3.5) and the $Q$ 's tend to be large.

The experimenter met the subjects for this study individually in a small, darkened room. The subjects were a miscellaneous group of college instructors, students, and townspeople, numbering 20 altogether. $^{3}$ The equipment consisted of two voice keys and a timeclock (100ths of a second) so set up that the experimenter set off the clock by speaking directly into his voice key and the subject stopped it by speaking the number (from 1-7) which indicated his judgment. The time on the clock indicated the period consumed by the subject in making a judgment. Time, then, is here considered as a measure of the ease of reaching a decision, the assumption being that stereotyped or polarized judgments will take less time than those over which the subject has to ponder.

The following instructions were read aloud to each subject:

Take a comfortable, relaxed position in the chair. The gadget you have in your hand is called a voice key. There is one just like it here in front of me. When I speak in mine a time clock is started. When you answer, it stops the clock. However, I want you to remember while taking this experiment that time is not the important factor. What we are interested in is your true response, your real opinion.

I will read you what we call a gradient, such as "kind ..... cruel." You are to assume that there are 7 steps between the two words. Thus, in this case, 1 would mean very kind; 2 would mean quite kind; 3, slightly kind; 4 means neither

${ }^{3}$ These subjects had not participated in the group study. 
kind nor cruel, no feeling either way, the middle; 5 would mean slightly cruel; 6 quite cruel; and 7 very cruel. Do you follow so far? (If negative reply, this paragraph was read over.)

After I read you such a gradient as "kind ..... cruel" I will give you another word, such as "Englishmen," and you are to indicate where you think "Englishmen" belong on the "kind . . . . . cruel" gradient by a number from 1-7. Thus, if you thought "Englishmen" were very kind you would say "1." If you thought they were somewhat cruel, you might say "5" or "6." Think of the 1 end of the gradient as always being nearest the first word of the pair and the 7 end as nearest the last word of the pair.

Remember, we are not interested in the time. We are interested in the responses you make. I will give you several warm-up items and then go right on into the test items. You'd better hold the voice key about a hand's breadth from your mouth and speak in your normal way. All set?

The gradient was given in such a manner that it did not start the timer; the experimenter then leaned close to the voice key to speak the concept. The reasons for this arrangement were two-fold. First, reading gradients last (setting off the clock with them) would increase the time difference in presentation and thereby introduce possible errors in the data. Second, other experiments (11) have indicated the prevalence and pervasiveness of a frame of reference which may be called up by certain terms (i.e., a generally negative or unfavorable attitude toward Dictators, Russians, etc.). Speaking such a concept first would give an opportunity for such a frame of reference to determine or influence the time for that item. Naming a gradient, on the other hand, gives the subject no opportunity to set up a frame of reference as he does not know whether it will be, for example, Russians or Americans he will be judging on it.

After each item was judged by a subject (thereby stopping the time-clock), the experimenter noted down the position of the judgment (by a number from 1-7) and the time taken to make it.

\section{Results}

The central problem was to determine the extent and consistency of differences between items chosen as polarized and those chosen as spread. Each of the 20 subjects gave a judgment on each of the 10 items and the time consumed in making these judgments was 
noted. From these data the average time taken for reaching a decision on the five polarized and the five spread items could be determined for each subject. Of the 20 subjects, 17 showed positive differences (polarized judgment-times shorter than spread), one subject showed no difference, and two showed negative differences (polarized longer than spread). The mean time for polarized items for all subjects was 2.53 seconds, while the mean time for spread items was 3.17 seconds.

The critical ratio for this difference between the means of polarized and spread items was 3.00. It was noted, however, that the data were correlated; that is, subjects differed considerably in their general judgment-time, hence some subjects would be relatively long in judging both polarized and spread items, yet still show positive differences between the two. The correlation of the average judgment-times by subjects between polarized and spread items was +.69 . When a correction for this is applied to the critical ratio given above, it becomes 5.33, which is entirely beyond what could be expected by chance.

It seems reasonable, therefore, to conclude that we have demonstrated a real difference in ease of judgment determined by the nature of the material. And as the nature of the materials for judgment was ordered in terms of extreme degrees of polarization, it may be concluded that polarization of certain patterns of thought in the culture is related to increased ease of judgment for individuals in the culture, on these same patterns of thought.

We may now turn to an item by item analysis of the data. In Table 1 , under $M$ are given the mean times taken by all 20 subjects in making judgments on each of the 10 items. The items are here ordered according to mean time, and the polarized items are indicated by asterisks. A theoretically perfect ordering would have been for the five polarized items to rank 1-5 and the five spread items, 6-10. As it is, there is only one shift from this theoretical expectation. Item 4, an item picked as spread, is somewhat out of position.

It will be well at this point to analyze the reason for the displacement of Item 4, Dictator, strong-weak. Superficially this item, referring to the group data in Table 1 , shows no clear differences from the other spread items; the median for the adult group on this item is only $3.7, .2$ of a step from the theoretical middle of the scale (3.5). It will be noted, however, that the $Q$, or measure 
of spread, is the largest of any item. The actual frequencies for the seven positions on the scale for this item were as follows:

DICTATOR: strong $25: 7: 9: 8: 9: 11: 19$ weak

This frequency distribution designates this item as bi-polar in nature. Thus, if subjects consider a dictator strong, he is felt to be very strong; on the other hand, if they think a dictator weak (probably through a secondary relationship of "weak" with "bad," dictators being definitely bad in the minds of nearly all our subjects), he is very weak. An adequate explanation of the relatively shorter time in judging this item would seem to be that this is really a polarized reaction having a bi-polar or dichotomous form.

The measure of difference, $D$, in the group data probably gives the most accurate single measure of polarization (excepting Item 4). This measure is the difference between the obtained median for the group and the theoretical mid-point of the 7-point scale used. Thus Item 1 , with a median of 6.0 , is 2.5 steps above 3.5 , the theoretical mid-point. Referring to the items designated as polarized (starred), it may be seen that the differences range from 1.7 up to 2.9. Of course, the medians may be either toward the favorable or unfavorable extremes ( 7.0 or 0.0 ) of the gradients. The spread items (unstarred), on the other hand, cluster closely about the theoretical mid-point of the scales, with differences ranging from .1 to .4. The rank-difference correlation of $D$-values with mean judgment-times is +.68 . These differences, then, give a measure of how extreme certain patterns of thought are in the culture.

$Q$-values offer a slightly different measure of polarization. They indicate how much variability there is of individual opinions about the group median. This is probably not as precise a measure of polarization as the $D$ because some judgments may be low on variability simply because discrimination is difficult or impossible. Item 8 is an example of this. The adult group, very reasonably, placed foreigners-in-general in the middle of the gradient, yet the $Q$ of 1.4 is as low as two of the polarized items. In general, however, the $Q$-values follow the trend shown by the $D$-values. The range for $Q$-values on polarized items is from .3 to 1.5 (slight variability) and for spread items is from 1.4 to 2.4 (greater variability). Similar to the relationship above, though smaller, the correlation of $Q$-values with mean judgment-times is +.44 . 
Having demonstrated the presence of significant differences between polarized and spread items lumped into groups, we may consider the significance of differences between specific items. As in the lumped data, it is permissible to introduce a correction for the systematic variation of individual differences in general judgmenttime. It will be remembered that the correlation of the average polarized and spread items by subjects was .69. Naturally, the correlations of single items with single items would be less. It was felt that, instead of working out the 90 -odd correlations to determine the $r$ of each item with every other item, taking the average of four such correlations would give a sufficiently reliable correction to apply to the critical ratios between any items. The correlations computed were between Items 1 and 7,2 and 9,5 and 10, and 6 and 8 . Each was between a polarized and a spread item, for these were the critical ratios to be determined. The average $r$ was .41 and this was used in correcting the critical ratios of all differences.

The two final columns in Table 1 present samples of these data, the critical ratios for the items indicated and the chances in 100 of such obtained differences being significant. Although these differences are not as strikingly significant as that between average polarized and spread items $(C R=5.33)$, they are in the same direction and certainly support the same conclusion: that items picked as polarized yield significantly shorter judgment-times than items picked as spread.

Item 4, the exceptional item, was discussed above. Critical ratios between it and the other items were not determined.

The critical ratio between Item 1, the polarized item with the shortest judgment-time, and Item 7, the spread item with the shortest judgment-time, was found to be $3.0(99+$ chances in 100 of significance). It is therefore unnecessary to determine $C R$ 's between Item 1 and the other spread items as they would all be greater than 3.0. This same procedure was followed with respect to the other differences.

There are significant differences between Item 1 and all spread items. This is also true for Item 2. The critical ratio between 3 and 7 is only 1.4 (92 in 100), but between 3 and the other spread items there are significant differences. The same statements hold for Item 5 as for Item 3 . There is practically no difference between Item 6 , the polarized item with the longest judgment-time, and 
Item 7 , the spread item with the shortest judgment-time, but the differences between 6 and the other spread items are fairly significant. These item-by-item comparisons, then, amply support the results of the comparison of lumped polarized and spread items.

The data also yield information on the relation of ease of judgment to the position (1-7) picked on the gradient. A possible theory of the use of gradients in thinking would be that extremes or polarities, being the simplest "all-or-nothing" type of reaction, would tend to have the shortest judgment-times; the middle of the scale, a secondary level of discrimination-neither one nor the other extreme-would tend to have a moderately short judgment-time; while other positions on a seven-point scale, calling for more discrimination, would show longer judgment-times than either the extremes or the middle of the gradient.

The data bear this theoretical position out. Figure 1 compares such a theoretical curve (dash-line) for a seven-point gradient with the obtained results (full line). The obtained points are based on

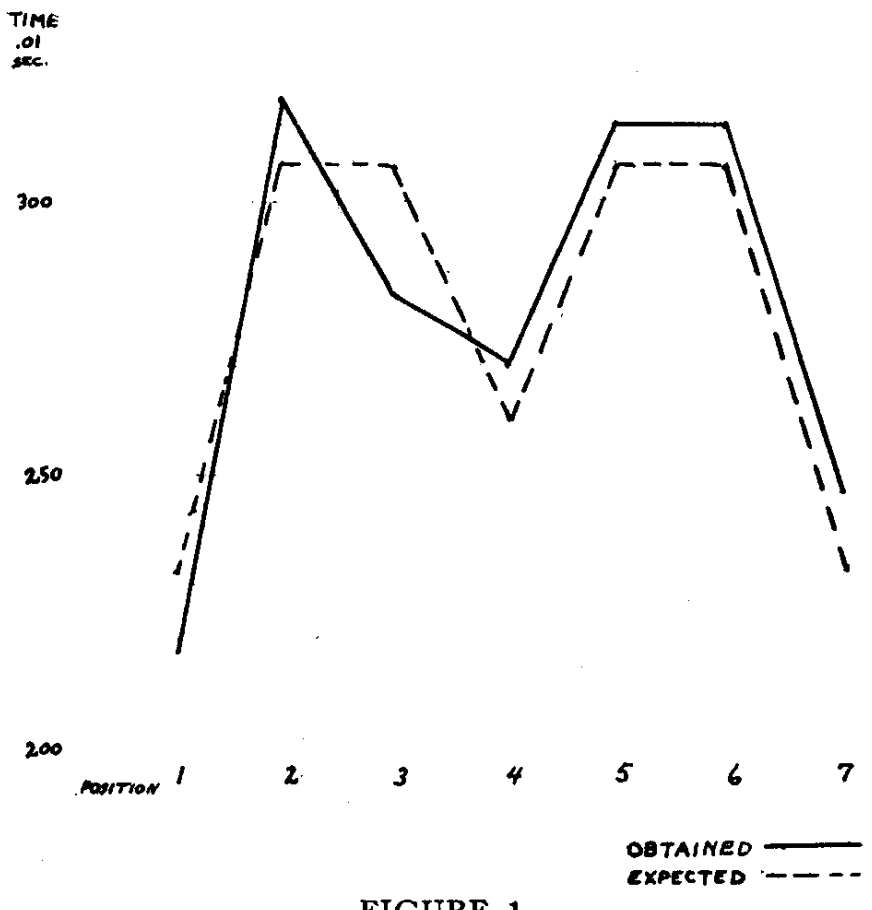

FIGURE 1 
the means of the judgment-times for all subjects using each position on the gradient. Thus Position 1 was called 28 times during the entire experiment (all 10 gradients included) and the mean time for these 28 judgments was $2.18 \mathrm{sec}$. Position 2 was used 28 times also, and the mean time was $3.29 \mathrm{sec}$, etc. Considering the fact that only 20 subjects were used in the experiment (for a total of 200 judgments), the obtained curve fits the theoretical expectation rather well..$^{4}$

Indeed, Position 3 is the only point far out of line. Analysis of individual subjects suggests the reason for this. One subject used Position 3 in five of his ten judgments and did not use Position 4 at all. It happens that this subject had the shortest mean judgmenttime of all subjects, only $1.59 \mathrm{sec}$. As there were only a total of 31 uses of Position 3, these five very short times by one subject, accounting for $1 / 6$ of the judgments for the position, tend to lower the mean time for this position unduly, but do not affect Position 4 in an equivalent fashion.

Table 2 gives the mean, $S D$ and $S D_{M}$ for each of the seven positions. The important critical ratios and chances in 100 of their significance are given. By "important critical ratios" we mean those which are for differences between the extremes and the middle of the scale, the extremes and the discrimination positions $(2,3,5,6)$, and between the middle and the discrimination points.

As to the first condition, the critical ratio between 1 and 4 is 2.5 (99 chances in 100 of significance), but that between 7 and 4 is only 1.2 ( 88 chances in 100 of significance). Critical ratios between the extremes or polarities (Positions 1 and 7 ) and the discrimination points $(2,3,5$, and 6$)$ are all quite high, ranging from 94 to 100 chances in 100 of being significant. Ratios between the middle position, 4, and discrimination Positions 2, 5, and 6 are also quite

\footnotetext{
${ }^{4}$ It would appear that these results are not in complete conformity with those of Cantril (4), who found that persons favorable to a certain value gave free associations to stimuli relevant to that value more rapidly than persons not favorable to it. Our findings indicate only slight differences for Positions 1-3 (favorable) as opposed to Positions 5-7 (unfavorable). The $C R$ for this difference is approximately .5 , although it is in the direction indicated by Cantril. Figure 1 shows that the mean time for Position 1 is somewhat lower than for Position 7, which also suggests same conclusion. The factor of intensity, however, appears to be crucial, and on this point Cantril's data are not complete enough for us to judge whether a real conflict in the two sets of data exists.
} 
TABLE 2

Results-BY Positions

\begin{tabular}{|c|c|c|c|c|c|}
\hline Position & Mean & $S D$ & $S D_{M}$ & $C R$ & Chs. $/ 100$ \\
\hline 1 & 218 & 75 & 14 & $\begin{array}{l}1+4=2.5 \\
1+2=4.5 \\
1+3=2.8 \\
1+6=3.3\end{array}$ & $\begin{array}{r}99 \\
100 \\
99 \\
100\end{array}$ \\
\hline 2 & 329 & 108 & 20 & & \\
\hline 3 & 284 & 108 & 19 & & \\
\hline 4 & 270 & 89 & 15 & $\begin{array}{l}4+2=2.3 \\
4+3=.6 \\
4+5=1.8 \\
4+6=1.5\end{array}$ & $\begin{array}{l}99 \\
73 \\
96 \\
93\end{array}$ \\
\hline 5 & 313 & 89 & 18 & & \\
\hline 6 & 313 & 137 & 25 & & \\
\hline 7 & 246 & 70 & 14 & $\begin{array}{l}7+2=3.5 \\
7+3=1.6 \\
7+4=1.2 \\
7+6=2.3\end{array}$ & $\begin{array}{r}100 \\
94 \\
88 \\
99\end{array}$ \\
\hline
\end{tabular}

reliable, ranging from 93 to 99 chances in 100 of being significant differences. Indeed, the only difference which shows no significance is between Positions 3 and 4, and this may be explained by the displacement of Position 3 due to the cause cited above.

The determined fact that "all-or-none" positions have shorter judgment-times than discrimination positions, coupled with the fact that polarized items have shorter judgment-times than spread items, suggests a common factor behind both results. Although further experimentation will be necessary to determine whether these two factors are independent or not, the data in this study strongly indicate that the position and item factors are independent. That is, the shorter judgment-times for Positions 1 and 7 are not responsible for the lowered judgment-times on the polarized items, or vice versa.

The basis for this conclusion lies in a scattergram which plots the positions chosen, item by item. If the two factors were closely related, it would be expected that the polarized items would show a definite preponderance of 1 and 7 judgments, while the spread items should show a preponderance of $2,3,5$, and 6 judgments. This is not the case. In fact, Item 1 , that having the shortest mean judg- 
ment-time, shows no uses of either Position 1 or 7 . Items 2 and 3 do have a preponderance of 1's or 7's, but most items show considerable variability of choice.

It would be expected that polarized items should show an excess of extreme judgments. It was fortunate for the purposes of this study that our subjects did not follow this tendency exactly. And it is of especial interest, then, that differences between polarized and spread items are as significant as they are. The results indicate the influence on judgment-time of both a position-on-the-gradient factor and a meaning factor, but the results do not offer a clear solution of the problem of the relation between these factors.

\section{Theoretical Discussion}

Items chosen as polarized and spread in a cultural sample show significant differences in the ease of judgment on them by individuals taken from that culture. This is particularly important in view of the fact that the 20 individual subjects did not invariably pick the positions on the gradients which were predominant in the group data. How, then, does it happen that judgment-times for polarized items, subject by subject and item by item, were lower than judgment-times for spread items, regardless of whether the subjects' position choices were polarized or not?

The fact of polarization of certain patterns of thought (as defined above) in the culture itself indicates that large numbers of people have formed definite opinions on these matters, have taken sides, and have become emotionally set. It is likely, then, that individuals drawn from the culture will likewise have formed opinions, taken sides, and become emotionally set on these same patterns of thought. These individuals have already gone through the judgment process, probably frequently in such patterns as the lowness of dictators and the foolishness of fighting. Hence the experimental situation finds them already "set" for these judgments. Whether these individuals' decisions are like those indicated by the group distribution or not, they have formed opinions-therefore their judgment-times will be shorter no matter what the position picked on the gradient.

In the case of items spread in the culture, however, the situation is different. The fact of "spreadness" or lack of crystallization of such thought patterns may indicate one of two things: $(a)$ that this pattern of thought has not become focused in the culture, has not 
been an object of discussion, and hence individuals are forced to improvise judgments on it, or (b) that it is still in the controversial stage-a variety of opinions are being set forth, but none is predominant-hence there is confusion about such a pattern of thought, and the individual in the experimental situation "muddles through" to his decision. Such an explanation may be seen to apply easily to such judgments as whether socialists are happy (Item 7), foreigners are tolerant (Item 8); or isolation is brave (Item 9).

A recent paper by Stagner (14) suggested that these two factors account for the failure of certain items on a scale for nationalistic attitudes to correlate highly with more widely discussed phases of national policy. The subjects had adopted a consistent "set" with regard to tariffs, armaments, and patriotic education, but not with regard to a rarely-mentioned proposal, a "United States of the World," or a highly controversial one, joining the League of Nations.

Johnson (6) has recently published a study in which he demonstrated a rather close relationship between the percentage of a group endorsing a given attitude statement and the confidence with which individuals endorse the statement. As in our own subjects, this relationship held even if the subjects did not conform to the majority opinion (i.e., the members of a small minority held their opinions more confidently than members of a relatively large minority). The same two factors might account for his findings. It may be suggested in passing that confidence is undoubtedly involved in judgment-time, although the causal relationship is not immediately obvious.

The problem of the meaning of words may be seen at the basis of differences in judgment-time in certain items. The bi-polar item concerning the strength or weakness of dictators comes into this category. The item with the longest judgment-time, the idealism or realism of a big navy, may also belong here. In this case the length of judgment-time (as well as the variability of the cultural sample) may be due to an inherent difficulty and vagueness in the words "idealistic" and "realistic." In some frames of reference "idealistic" connotes a favorable opinion, while in others "realistic" is the good value. An upright, virtuous young man is "idealistic," yet he is expected to be "realistic" about the problems of life.

The relative specificity of the various concepts used in the experiment may also affect judgment-time. Concepts like Dictator, Isolation, and Big Navy seem to be reacted to in a much more specific 
fashion than such concepts as Socialists and Foreigners. In fact, the concentration of judgments on Position 4 for the Foreigner item indicates an awareness on the part of the subjects of the meaningless generality of the concept.

Within the limits of the present experiment two relatively independent factors influencing judgment-time have been indicated: $(a)$ a position-on-the-gradient factor, possibly a function of the thinking process itself, and $(b)$ an item-meaning factor, presumably a function of the meaning gained by the subject from the concept and gradient labels and their interrelationship in his mind.

The fact that thinking in terms of extremes of a gradient is easier (or occurs more readily) than in terms of finer discriminations on the gradient may well be related to a similar fact observed in rote learning studies. It has been found, in the latter case, that learning of the beginning and end of a series is swifter than the learning of items in the middle of the series. In fact, an experiment in which the total series was broken in the middle (13) showed that learning was most efficient at the extremes of the entire series and fairly efficient around the break in the middle of the series, a result which shows striking similarity to the use of gradients in thinking as demonstrated here (see Figure 1). Rating scale studies of personality indicate also the tendency to use extreme judgments more frequently and easily than intermediate steps on the scale.

When sufficient studies on these points have been analyzed in relation to one another, it may be possible to describe what might be termed a (Gestalt) theory of thinking in terms of the psychological distinctiveness of the material. Apparently, the ends of a series are psychologically easily discriminable from the rest of the environment and from the remainder of the gradient. Other points in a series or gradient which are given special significance (i.e., made to function as "figure" rather than "ground"), as in the "broken" series above, also become psychologically more easily discriminable, therefore simpler to deal with during the thinking process.

Three levels of difficulty in thinking in terms of gradients are suggested in the present experiment. The simplest level, as measured by judgment-time, is in terms of extremes. A further discrimination serves to bring the middle position into prominence. And the third and most difficult level of thinking is when finer distinctions still are made. Examination of the papers of individual subjects 
in the group studies cited above indicates that some people mark gradients almost exclusively in terms of 1 and 7 ; some use only 1, 4, and 7; while others employ the entire scale. Preliminary analysis of these data indicate that these differences are related to occupation, education, and intelligence, the more critical thinkers making a more discriminatory use of the entire scale.

The term "judgment-time" has been used consistently in this paper to denote that time which passes between the presentation of the judgment situation to the subject and his solution or decision. Obviously, judgment-time, as used here, is closely related to both simple reaction-time and association-time. The simple reaction-time is a part of what we are terming judgment-time, although it is necessarily a small and, as far as individual differences are concerned, unimportant part. Judgment-time may be correctly considered as a special and rigidly controlled type of association-time in which the direction and nature of the association is indicated for the subject completely, his only task being to indicate the degree of relationship. Judgment-times may be expected to show more meaningful and significant relations to intellectual and personality factors than simple reaction-times, for a great many, if not most, of the adjustments in life involve judgment situations as defined here (7).

\section{REFERENCES}

1. Allport, F. H. Social Psychology. Boston: Houghton-Mifflin, 1924.

2. The J-curve hypothesis of conforming behavior. J. Soc. Psychol., 1934, 5, 141-183.

3. Arnold, T. The Folklore of Capitalism. New Haven: Yale Univ. Press, 1937.

4. Cantril, H. General and specific attitudes. Psychol. Monog., 1932, 42, No. 192.

5. Healy, W. Bronner, A. F., \& Bowers, A. M. The Structure and Meaning of Psychoanalysis. New York: Knopf, 1930.

6. Johnson, D. M. Confidence and the expression of opinion. J. Soc. Psychol., S.P.S.S.I. Bull., 1940, 12, 213-220.

7. Karwoski, T. F., Odbert, H. S., \& Osgood, C. E. Studies in synesthetic thinking: II. The rôle of form in visual responses to music. J. Gen. Psychol. (To appear.)

8. Lynd, R. S., \& Lynd, H. M. Middletown in Transition. New York: Harcourt, Brace, 1937.

9. Metressel, M. The all-or-none nature of emotional thinking. $J$. of Psychol., 1940, 9, 323-326. 
10. Mumford, L. Technics and Civilization. New York: Harcourt, Brace, 1934.

11. Oscood, C. E., \& STAGNer, R. Analysis of a prestige frame of reference by a gradient technique. J. Appl. Psychol., 1941, 25, 275-290.

12. Stagner, R., \& OsGoOd, C. E. An experimental analysis of a nationalistic frame of reference. J. Soc. Psychol., 1941, 14, 389-401.

13. StAGner, R. Factors influencing memory value of words in a series. J. Exper. Psychol., 1933, 16, 129-137.

14. - A correlational analysis of nationalistic opinions. J. Soc. Psychol., S.P.S.S.I. Bull., 1940, 12, 197-212.

Department of Psychology

Yale University

New Haven, Connecticut 